\title{
D-STAG: a Formalism for Discourse Analysis based on SDRT and using Synchronous TAG
}

\author{
Laurence Danlos \\ Université Paris Diderot, IUF, ALPAGE \\ Laurence.Danlos@linguist.jussieu.fr
}

\begin{abstract}
We propose D-STAG, a new formalism for the automatic analysis of discourse. The analyses computed by D-STAG are hierarchical discourse structures annotated with discourse relations, which are compatible with discourse structures computed in SDRT. A discursive STAG grammar pairs up trees anchored by discourse connectives with trees anchored by (functors associated with) discourse relations.
\end{abstract}

\section{Introduction}

We propose a new formalism for the automatic analysis of texts, called D-STAG for Discourse Synchronous TAG. This formalism extends a sentential syntactic and semantic analyzer to the discursive level: a discursive analyzer computes the "discourse structure" of the input text. Discourse structures consist of "discourse relations" (also called "rhetorical relations") that link together discourse segments - or more accurately, the meanings these discourse segments convey. A discourse is coherent just in case every proposition that is introduced in the discourse is rhetorically connected to another bit of information, resulting in a connected structure for the whole discourse.

For the discursive part of our analyzer, we rely on SDRT - Segmented Discourse Representation Theory [1,2]. D-STAG computes discourse structures which are compatible with those produced in SDRT. Therefore, D-STAG can take advantage of the results brought by this discourse theory.

The research done in the framework of SDRT is theory-oriented, providing formally detailed accounts of various phenomena pertaining to discourse. Much less focus has been put on the issue of implementing a robust and efficient discourse analyzer. For this aspect of the work, we have designed a formalism based on TAG - Tree Adjoining Grammar [3]. After being used successfully for syntactic analysis in various languages, TAG has been extended in two directions: moving from sentential syntactic analysis to semantic analysis - with, among others, STAG [4-6] -, and moving from the sentence level to the discourse level. The discourse level initially focuses on text generation - with, among others, G-TAG [7] —, then on discourse parsing — with, among others, D-LTAG [8]. The new formalism presented here relies on all this previous work. In particular, DSTAG and D-LTAG, which have roughly the same goal, share the same architecture with three components: 
1. a sentential analyzer, which provides the syntactic and semantic analyses of each sentence in the discourse given as input,

2. a sentence-discourse interface, which is a mandatory component if one wants not to make any change to the sentential analyzer,

3. a discursive analyzer, which computes the discourse structure.

This paper is organized as follows. Section 2 presents the main discursive linguistic data that motivate D-STAG. Section 3 gives an introduction to STAG. Section 4 briefly describes the sentence-discourse interface. Section 5 explains in detail the discursive analyzer. Section 6 compares D-STAG and D-LTAG.

\section{Discursive Linguistic Data}

A discourse relation is often explicitly expressed by a "discourse connective". The set of discourse connectives includes subordinating and coordinating conjunctions (because, or) and discourse adverbials (next, therefore). Connectives can be ambiguous. For example, then lexicalizes the relation Narration in a narrative (Fred went to the supermarket. Then, he went to the movies.) whereas it lexicalizes Continuation in an enumeration ( ... The second chapter presents a state of the art. Then, the third chapter explains the problematics.). Discourse relations need not be explicitly marked. For example, the relation Explanation in the connective-free discourse Fred fell. Max tripped him up. of the form $C_{1} \cdot C_{2}$. must be inferred from (extra)linguistic knowledge. For such a case, we assume the existence of an empty adverbial connective, noted $\epsilon$, following a proposition made in [9]. So the previous discourse is assumed to be of the form $C_{1} . \epsilon C_{2}$., and we say, although somewhat inaccurately, that $\epsilon$ lexicalizes Explanation. In a nutshell, a discourse relation can be considered as a semantic predicate with two arguments which is lexicalized by a discourse connective (possibly empty) with two arguments. The arguments of a discourse relation/connective are the discursive semantic/syntactic representations of the same (continuous) discourse segments, These are the basic principles on which our STAG discursive grammar relies.

One should wonder what discourse structures correspond to when represented as dependency graphs (in which a predicate dominates its arguments). The idea is widespread that dependency graphs representing discourse structures are treeshaped: this is a basic principle in RST - Rhetorical Structure Theory [10,11] -, a theory on which many text generation or parsing systems have been based for the last twenty years. This is also a principle which guided the conception of D-LTAG. Yet this tree-shaped structure is more a myth than a reality, as shown in [12] and in some of our previous work [13,14]. SDRT discourse structures are not represented as dependency graphs, however, in our two aforementioned papers and in this one, we convert SDRT discourse structures into dependency graphs. These dependency graphs are DAGs - Directed Acyclic Graphs - which are not necessarily tree-shaped. However, these DAGs respect strong constraints which rule out a number of DAGs that don't correspond to any discourse structure. We shall justify this claim with the discourses of the form $C_{1}$ because $C_{2} . A d v_{2} C_{3}$., 
in which because lexicalizes Explanation and $C_{i}$ symbolizes the $i$ th clause (the logical form for $C_{i}$ is noted $F_{i}$ ). These discourses yield four types of interpretation — but no more than four — which are illustrated in examples (1). ${ }^{1}$

(1)a. Fred is in a bad mood because he lost his keys. Moreover, he failed his exam.

b. Fred is in a bad mood because he didn't sleep well. He had nightmares.

c. Fred went to the supermarket because his fridge is empty. Then, he went to the movies.

d. Fred is upset because his wife is abroad for a week. This shows that he does love her.

In (1a), $A d v_{2}=$ moreover lexicalizes the relation Continuation. The discourse segment $C_{2} A d v_{2} C_{3}$ forms a complex constituent whose logical form, i.e. Continuation $\left(F_{2}, F_{3}\right)$, is the second argument of Explanation. So the discourse structure is Explanation $\left(F_{1}\right.$, Continuation $\left.\left(F_{2}, F_{3}\right)\right)$, which corre- $F_{1}$ sponds to a tree-shaped dependency DAG, see the adjacent figure with $R_{1}=$ Explanation and $R_{2}=$ Continuation. In this example, the second discursive argument of the conjunction because crosses a sentence boundary.

In (1b), $A d v_{2}=\epsilon$ lexicalizes Explanation. The discourse structure is $\operatorname{Explanation}\left(F_{1}, F_{2}\right) \wedge$ Explanation $\left(F_{2}, F_{3}\right)$, which corresponds to a non treeshaped dependency DAG.

In (1c), $A d v_{2}=$ then lexicalizes Narration. The discourse structure is Explanation $\left(F_{1}, F_{2}\right) \wedge$ $\operatorname{Narration}\left(F_{1}, F_{3}\right)$, which corresponds to a non treeshaped dependency DAG.

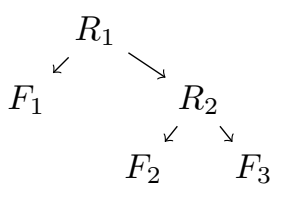

In (1d), $A d v_{2}=\epsilon$ lexicalizes Commentary. The discourse segment $C_{1}$ because $C_{2}$ forms a complex constituent whose logical form is the first argument of Commentary. So the discourse structure is Commentary $\left(\right.$ Explanation $\left.\left(F_{1}, F_{2}\right), F_{3}\right)$, which corre- $F_{1}$ sponds to a tree-shaped dependency DAG.
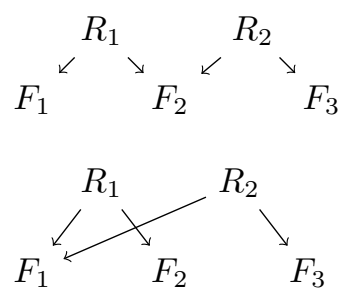

In conclusion, these empirical data show that a formalism for the automatic analysis of discourse must be able to compute dependency structures which are not tree-shaped. ${ }^{2}$ This principle guided the conception of D-STAG. More precisely,

${ }^{1}$ In [15], these four types of interpretation are finally brought to light, thanks to the Penn Discourse Tree Bank (PDTB), which is an English corpus manually annotated for discourse relations and their arguments [16].

${ }^{2}$ In RST, (1b) and (1c) are represented as trees that must be interpreted with the "Nuclearity Principle" [17]. However, as explained in [14], the Nuclearity Principle leads to a wrong interpretation for (1d), namely Explanation $\left(F_{1}, F_{2}\right) \wedge \operatorname{Commentary}\left(F_{1}, F_{3}\right)$. 
from this (and other) data, we laid down the constraints below which govern the arguments of a discourse connective/relation using the following terminology. The clause in which a connective appears is called its "host clause". An adverbial connective appears in front of its host clause or within its VP. A subordinating conjunction always appears in front of its host clause, which is called an "adverbial clause." At the sentence level, an adverbial clause modifies a "matrix clause." It is located on its right, on its left, or inside it before its VP. When it is located on its right, the subordinating conjunction is said to be "postposed," otherwise it is said to be "preposed." A discourse connective/relation has two arguments which are the syntactic/semantic representations of two discourse segments that we call the "host segment" and the "mate segment". These segments are governed by the following constraints.

Constraint 1 The host segment of a connective is identical to or starts at its host clause (possibly crossing a sentence boundary).

Constraint 2 The mate segment of an adverbial is anywhere on the left of its host segment (generally crossing a sentence boundary). ${ }^{3}$

Constraint 3 The mate segment of a postposed conjunction is on the left of its host segment without crossing a sentence boundary.

Constraint 4 The mate segment of a preposed conjunction is identical to or starts at the matrix clause (possibly crossing a sentence boundary).

\section{Introduction to TAG and STAG}

This section is reproduced except where noted from [6] with permission of the authors. It begins with a brief introduction to the use of TAG in syntax.

"A tree-adjoining grammar (TAG) consists of a set of elementary tree structures and two operations, substitution and adjunction, used to combine these structures. The elementary trees can be of arbitrary depth. Each internal node is labeled with a nonterminal symbol. Frontier nodes may be labeled with either terminal symbols or nonterminal symbols and one of the diacritics $\downarrow$ or $*$. Use of the diacritic $\downarrow$ on a frontier node indicates that it is a substitution node. The substitution operation occurs when an elementary tree rooted in the nonterminal symbol $A$ is substituted for a substitution node labeled with the nonterminal symbol $A$. Auxiliary trees are elementary trees in which the root and a frontier node, called the foot node and distinguished by the diacritic $*$, are labeled with the same nonterminal. The adjunction operation involves splicing an auxiliary tree with root and designated foot node labeled with a nonterminal $A$ at a node in an elementary tree also labeled with nonterminal $A$. Examples of the substitution and adjunction operations on sample elementary trees are shown in Figure 1."

"Synchronous TAG (STAG) extends TAG by taking the elementary structures to be pairs of TAG trees with links between particular nodes in those trees. An

\footnotetext{
${ }^{3}$ However, the mate segment must conform to the Right Frontier Constraint, which has been postulated in SDRT, see Sect. 4.1.1.
} 

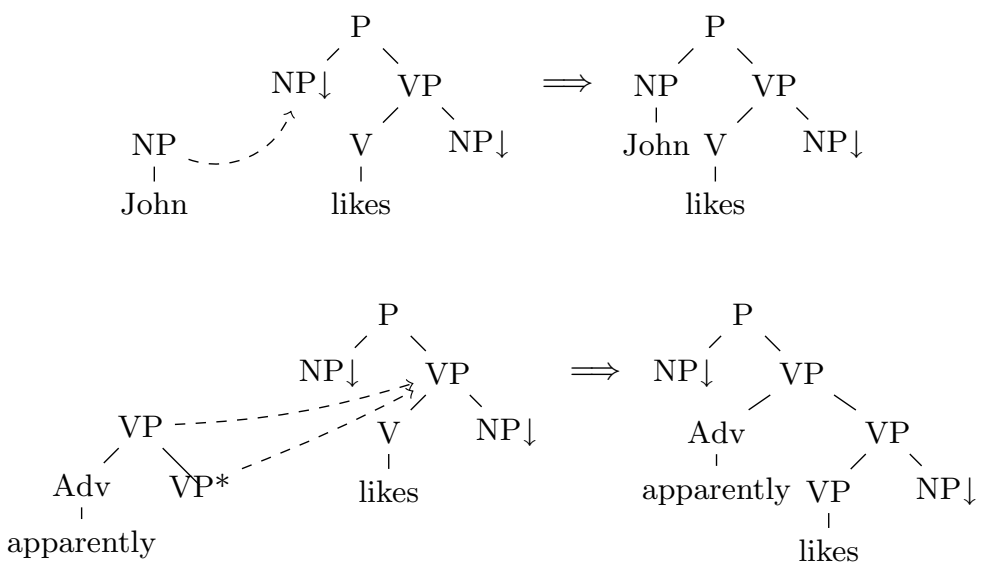

Fig. 1. Example TAG substitution and adjunction operations (reproduced from [6])

STAG is a set of triples, $\left\langle t_{L}, t_{R}, \frown\right\rangle$ where $t_{L}$ and $t_{R}$ are elementary TAG trees and $\frown$ is a linking relation between nodes in $t_{L}$ and nodes in $t_{R}[4,5]$. Derivation proceeds as in TAG except that all operations must be paired. That is, a tree can only be substituted or adjoined at a node if its pair is simultaneously substituted or adjoined at a linked node." We notate the links by using circled indices (e.g. (1) marking linked nodes.
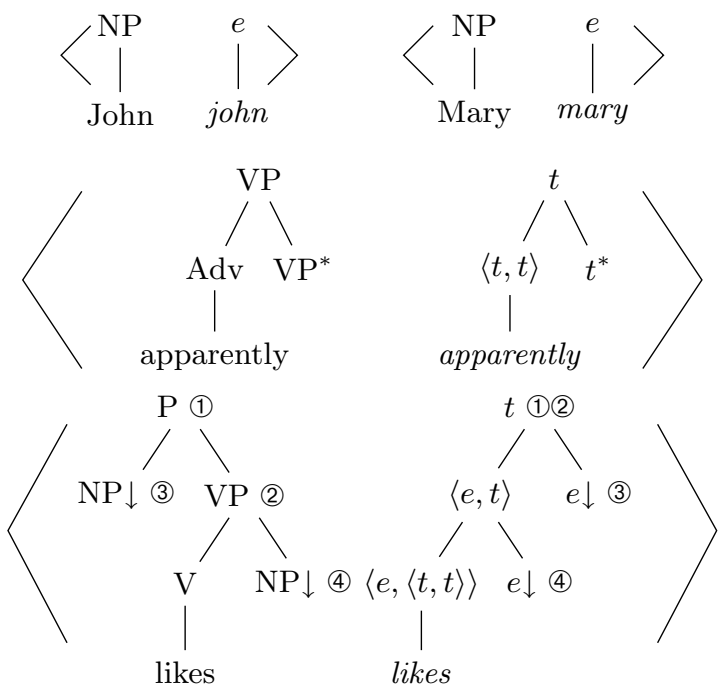

Fig. 2. An English syntax/semantics STAG fragment (reproduced from [6]) 
STAG has been successfully used in an English sentential syntax/semantics interface [6]. For the sentence John apparently likes Mary, Fig. 2 gives the STAG fragment, Fig. 3 the derivation tree and the derived tree pair. In derivation trees, "substitutions are notated with a solid line and adjunctions are notated with a dashed line. Note that each link in the derivation tree specifies a link number in the elementary tree pair. The links provide the location of the operations in the syntax tree and in the semantics tree. These operations must occur at linked nodes in the target elementary tree pair. In this case, the noun phrases John and Mary substitute into likes at links (3) and (4) respectively. The word apparently adjoins at link (2). The resulting semantic representation can be read off the derived tree by treating the leftmost child of a node as a functor and its siblings as its arguments. Our sample sentence thus results in the semantic representation apparently(likes(john, mary))."

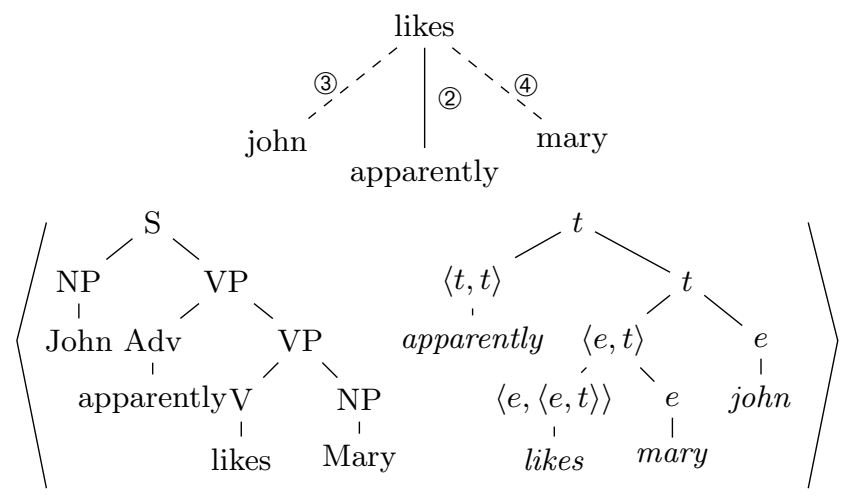

Fig. 3. Derivation tree and derived tree pair for John apparently likes Mary (reproduced from [6] )

\section{Sentence-Discourse Interface}

We first explain why this interface is necessary. The idea in D-STAG is to extend a sentential analyzer to the discourse level without making any change to it. Yet, one cannot directly pass from sentence to discourse because there are mismatches between the arguments of a connective at the discourse level and its arguments at the sentence level. First, an adverbial connective has compulsorily two arguments at the discourse level, whereas it has only one argument at the sentence level. Second, a subordinating conjunction can have an argument at the discourse level which crosses a sentence boundary (see (1a) and (3) below), whereas this is out of the question at the sentence level.

In conclusion, it is necessary to pass through a sentence-discourse interface which gives sentence boundaries the simple role of punctuation signs and which 
allows us to re-compute the (two) arguments of a connective. Such an interface is also used in D-LTAG, by which we were inspired. From the sentential syntactic analysis, this interface deterministically produces a "Discourse Normalized Form" (henceforth DNF), which is a sequence of "discourse words:" a discourse word is mainly a connective, an identifier $C_{i}$ for a clause (without any connective) or a punctuation sign. The syntactic and semantic analyses for $C_{i}$ s are those obtained by the sentential analyzer by removing connectives. An adverbial connective is moved in front of its host clause if not already there, while keeping a trace of its original position. If a normalized sentence (except the very first one) doesn't start with an adverbial connective, the empty connective $\epsilon$ is introduced. As an illustration, for (2), the DNF is $C_{1} . \epsilon$ as $C_{2}, C_{3}$. then internal $C_{4}$ because $C_{5}$.

(2) Fred went to the movies. As he was in a bad mood, he didn't enjoy it. He then went to a bar because he was dead thirsty.

The sequence of discourse words making up a DNF follows a regular grammar. The discursive component of D-STAG is illustrated below with the following regular expressions. A DNF without any preposed conjunction follows the regular expression $C$ (Punct Conn $C)^{*}$, in which the sequence Punct Conn is either . Adv or (, ) $C o n j$ where the comma is optional. It is noted $C_{1} C_{o n n} C_{2} \ldots C_{n-1} C_{n}$ disregarding punctuation signs. A DNF with a preposed conjunction includes one element $C$ which is preceded by the expressions $\operatorname{Conj} C((,) \operatorname{Conj} C)^{*}$. Finally, $A d v$ and $C o n j$ can be optionally followed or preceded by a modifier (Sect. 5.2).

This regular grammar should be completed to take into account coordinating conjunctions. Moreover, it takes into account neither clausal complements nor incident clauses nor relative clauses, while these sub-clauses may play a role at the discourse level. We plan to complete the regular grammar for DNFs in future research and to extend the discursive component of D-STAG accordingly.

\section{Discursive Component of D-STAG}

For a clause $C_{i}$ (without any connective), the sentential-discourse interface provides its syntactic tree rooted in $S$ and noted $T_{i}$, its semantic tree rooted in $t$ and noted $F_{i}$, and its derivation tree noted $\tau_{i}$. To plug the clausal analyses into the discourse ones, we use the pair $\alpha S$-to- $D$ given in Fig. 4 -a. In the rest of this paper, we note $\tau_{i}^{\prime}$ the derivation tree made of $\alpha S$-to- $D$ in which $\tau_{i}$ is substituted at link $\odot ; \tau_{1}^{\prime}$ corresponds to the pair given in Fig. 4-b. We also use the following convention: as any tree of our grammar includes at the most one substitution node, this one (when it exists) is systematically marked with link ๑.

Given the regular grammar for DNFs we have just presented, the discourse grammar includes trees anchored by a connective (co-anchored by a punctuation sign) and trees anchored by a connective modifier (Sect. 5.2).

\subsection{STAG Grammar for Connectives}

When a given connective $\operatorname{Conn}_{i}$ lexicalizes a single discourse relation $R_{i}$, the basic principle of the discursive STAG grammar consists in designing a tree pair, noted 


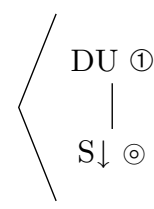

(a) $\alpha S-t o-D$

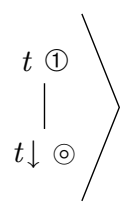

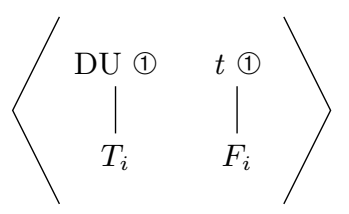

(b) $\tau_{i}^{\prime}$

Fig. 4. Tree pairs $\alpha S$-to- $D$ and $\tau_{i}^{\prime}$

$\operatorname{Conn}_{i} \div R_{i}$, whose syntactic tree is anchored by Conn $_{i}$ and whose semantic tree is anchored by a lambda-term associated with $R_{i}$. We say, although somewhat inaccurately, that the semantic tree is anchored by $R_{i}$. When a connective is ambiguous, i.e. it lexicalizes several discourse relations, it anchors as many syntactic trees as discourse relations it lexicalizes (this is in particular the case for the empty connective $\epsilon$ ). However, ambiguity issues are not in the scope of this paper.

We start with adverbials and postposed conjunctions, which are connectives with a similar behavior. Then, we move (Sect. 4.1.2) to preposed conjunctions.

\subsubsection{Adverbial Connectives and Postposed Conjunctions}

Syntactic trees. The syntactic trees anchored by an adverbial connective or by a postposed conjunction are given in Fig. 5, in which a discourse connective is of category $D C$. Disregarding the features for now, these trees differ only in the co-anchors which are punctuation signs of category Punct.
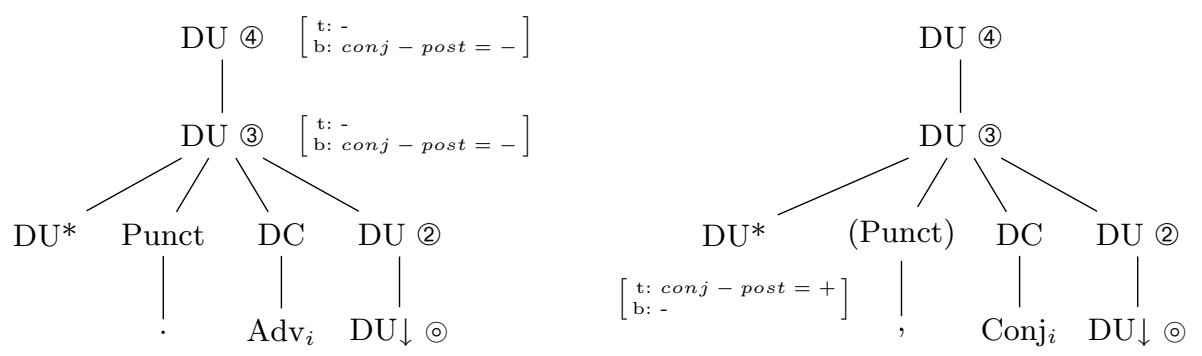

Fig. 5. Syntactic trees for adverbial connectives and postposed conjunctions

These trees observe the following principles: they are auxiliary trees with two arguments given by a substitution node $D U \downarrow$ and a foot node $D U^{*}$, where $D U$ (Discourse Unit) symbolizes the discursive syntactic analysis of a discourse segment. The substitution node is for the host argument of the connective, i.e. the $D U$ for the host segment substitutes at $D U \downarrow$. If the $D U$ for the host clause has undergone an adjunction, then the host segment starts at - but is not identical to - the host clause, see Constraint 1 in Sect. 2. The foot node corresponds to 
the mate argument of the connective. It is located on its left, which conforms to Constraints 2 and 3 . The fact that the mate segment of a postposed conjunction cannot cross a sentence boundary, contrarily to that of an adverbial, is handled with features $[\operatorname{conj}-$ post $= \pm]$ explained later.

In a DNF of the form $C_{1}$ Conn $_{1} C_{2}$ Conn $_{2} \ldots C_{n}$ (disregarding punctuation signs), the attachment of a new segment $\operatorname{Conn}_{n} C_{n+1}$ is only realized by adjunction of the tree anchored by $\operatorname{Conn}_{n}$ - in which the syntactic tree of the pair $\tau_{n+1}^{\prime}$ has been substituted - at a node of the syntactic tree representing $C_{1} \ldots C_{n}$. Keeping to the linear order of the DNF requires that the adjunction of a new segment be realized at a node which is located on the right frontier of the syntactic tree. That is the reason why the trees anchored by an adverbial or a postposed conjunction include three nodes labelled $D U$, with link (2), (3) or (4), on their right frontier. These nodes are marked with different links, which allows us to get various semantic interpretations, as shown below. There exist three nodes $D U$ with different links, and not a single node $D U$ with three different links, so as to allow several adjunctions to different nodes, for example an adjunction at $D U(3)$ to attach the new segment $C_{o n n} C_{n+1}$ and an adjunction at $D U(4)$ to attach the segment $C_{\text {onn }} n_{n+1} C_{n+2}$. It should be noted that if an adjunction is done at $D U(3)$ to attach $C o n n_{n} C_{n+1}, D U(2)$ is no longer on the right frontier of the syntactic tree. Therefore, $D U(2)$ can no longer be an adjunction site to attach Conn $_{n+1} C_{n+2}$. This constraint will be generalized in Constraint 5 below.

Semantic trees. At first sight, one could consider that a discourse relation $R_{i}$ is associated with the functor $\mathcal{R}_{i}=\lambda x y \cdot R_{i}(x, y)$ with $x, y: t, R_{i}(x, y): t$, and $\mathcal{R}_{i}:\langle t,\langle t, t\rangle\rangle, \mathcal{R}_{i}$ anchoring a tree with a foot node $t *$ and a substitution node $t \downarrow$. Yet this is appropriate only to analyze a simple DNF with two clauses, for example a DNF of the form $C_{1}$ because $C_{2}$ as shown in Fig. 6 in which $\beta_{1}=$ because $_{\text {post }} \div$ Explanation

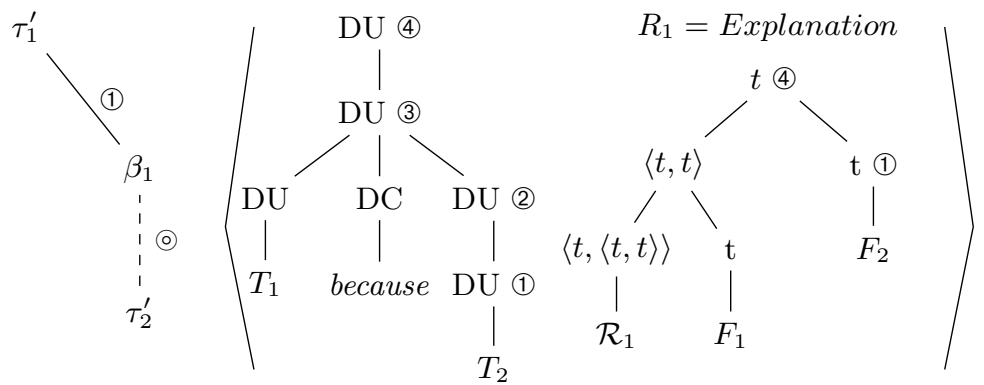

Fig. 6. Derivation tree and derived tree pair for a DNF of the form $C_{1}$ because $C_{2}$ (using the functor $\mathcal{R}_{1}$ in the semantic tree)

However, with this simple functor $\mathcal{R}_{i}$, it is impossible to obtain four interpretations (of which two are a conjunction of formulae) for DNFs with three clauses (see Sect. 2). Therefore, we define two type-shifting operators $\Phi^{\prime}$ and $\Phi^{\prime \prime}$ : they take $\mathcal{R}_{i}$ as argument and return two new functors $\mathcal{R}_{i}^{\prime}$ and $\mathcal{R}^{\prime \prime}{ }_{i}$ associated with the discourse relation $R_{i}$. 
Definition $1 \Phi^{\prime}=\lambda \mathcal{R}_{i} X Y \cdot X\left(\lambda x . Y\left(\lambda y \cdot \mathcal{R}_{i}(x, y)\right)\right)$

$\Phi^{\prime}\left(\mathcal{R}_{i}\right)=\mathcal{R}_{i}^{\prime}=\lambda X Y \cdot X\left(\lambda x . Y\left(\lambda y \cdot \mathcal{R}_{i}(x, y)\right)\right)$

with $X, Y: t t t=\langle\langle t, t\rangle, t\rangle$ and $x, y: t$

$\Phi^{\prime}$ triggers a type raising. The resulting functor $\mathcal{R}_{i}^{\prime}$ is of type $\langle t t t,\langle t t t, t\rangle\rangle$ in which $t t t$ symbolizes the type $\langle\langle t, t\rangle, t\rangle$. It co-anchors tree (A), given in Fig. 7-a, whose foot node is of type $t$. (A) is used for adjunctions at links (1) and (4). If the first argument of $\mathcal{R}^{\prime}{ }_{i}$ is $\lambda P . P\left(F_{1}\right)$ of type $t t t$, the second one $\lambda Q . Q\left(F_{2}\right)$ of type $t t t$, then the result is $R_{i}\left(F_{1}, F_{2}\right)$ of type $t$. So, for a DNF with two clauses, $\mathcal{R}^{\prime}{ }_{i}$ leads to the same result as $\mathcal{R}_{i}$. Yet, the type raising is necessary to introduce nodes $t t t$ (2) and $t t t$ (3) at which (B) can adjoin.

Definition $2 \Phi^{\prime \prime}=\lambda \mathcal{R}_{i} X Y P . X\left(\lambda x . Y\left(\lambda y \cdot \mathcal{R}_{i}(x, y) \wedge P(x)\right)\right)$

$\Phi^{\prime \prime}\left(\mathcal{R}_{i}\right)=\mathcal{R}^{\prime \prime}{ }_{i}=\lambda X Y P . X\left(\lambda x . Y\left(\lambda y \cdot \mathcal{R}_{i}(x, y) \wedge P(x)\right)\right)$

with $X, Y: t t t=\langle\langle t, t\rangle, t\rangle, P:\langle t, t\rangle$ and $x, y: t$

$\Phi^{\prime \prime}$ introduces a conjunction of terms. The resulting functor $\mathcal{R}^{\prime \prime}{ }_{i}$ is of type $\langle t t t,\langle t t t, t t t\rangle\rangle$. It anchors tree (B), given in Fig. 7-b, whose foot node is of type ttt. (B) is used for adjunctions at links (2) and (3). If the first argument of $\mathcal{R}^{\prime \prime}{ }_{i}$ is $\lambda P . P\left(F_{1}\right)$, the second one $\lambda Q . Q\left(F_{2}\right)$, then the result is $\lambda P .\left(R_{i}\left(F_{1}, F_{2}\right) \wedge P(F 1)\right)$ of type $t t t$.

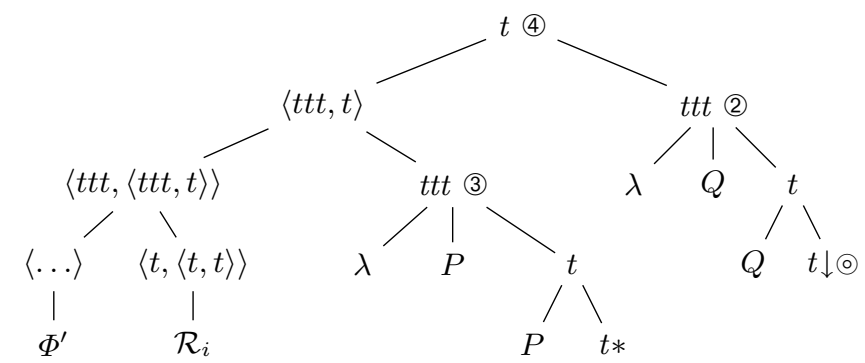

(a) Tree (A)

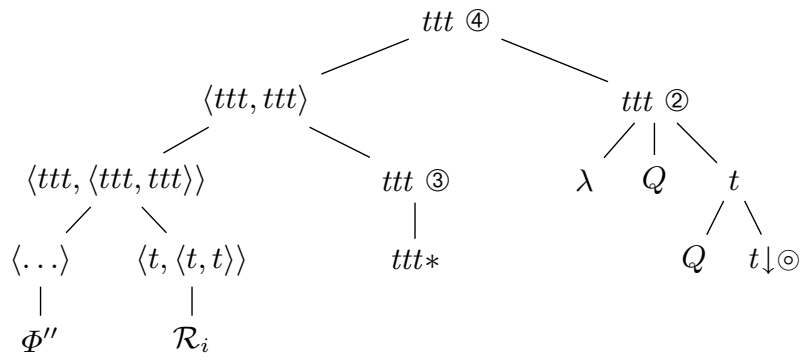

(b) Tree (B)

Fig. 7. Semantic trees (A) and (B) anchored by $\mathcal{R}_{i}^{\prime}=\phi^{\prime}\left(\mathcal{R}_{i}\right)$ and $\mathcal{R}^{\prime \prime}{ }_{i}=\phi^{\prime \prime}\left(\mathcal{R}_{i}\right)$ 
Analysis of DNFs with three clauses. For DNFs with three clauses, four types of interpretation must be computed. These were illustrated in examples (1) of the form $C_{1}$ because $C_{2} . A d v_{2} C_{3}$ in Sect. 2, and we are going to explain the analysis of these examples. We note $\beta_{1}$ the tree pair because post $\div$ Explanation and $\beta_{2}$ the pair $A d v_{2} \div R_{2}$. After analyzing $C_{1}$ because $C_{2}$, the syntactic tree is that shown in Fig. 6 . The right frontier of this tree includes four nodes labelled $D U$ which can receive the adjunction of the syntactic tree of $\beta_{2}$. These nodes are marked with link (1) coming from the syntactic tree of $\tau_{2}^{\prime}$ or link (2), (3) or (4) coming from the syntactic tree anchored by because. The analyses of the four examples in (1) are obtained by adjoining $\beta_{2}$ at one of these links.

We start with (1a) with $\beta_{2}=$ moreover $\div$ Continuation, for which the discourse structure is Explanation $\left(F_{1}\right.$, Continuation $\left.\left(F_{2}, F_{3}\right)\right)$. This is obtained by adjoining $\beta_{2}$ at link (1) of $\tau_{2}^{\prime}$. The node with link (1) in the semantic tree of $\tau_{2}^{\prime}$ is of type $t$. Therefore, one must use tree (A) anchored by $\mathcal{R}_{2}^{\prime}$, whose foot node is of type $t$. The semantic derived tree for (1a) is given in Fig. 8. The sub-tree rooted at Gorn address 2 results in $\lambda P . P\left(\right.$ Continuation $\left.\left(F_{2}, F_{3}\right)\right)$ with $P:\langle t, t\rangle$. So Continuation $\left(F_{2}, F_{3}\right)$ is the second argument of $R_{1}=$ Explanation, whose first argument is $F_{1}$, hence the formula Explanation $\left(F_{1}\right.$, Continuation $\left(F_{2}, F_{3}\right)$ ).

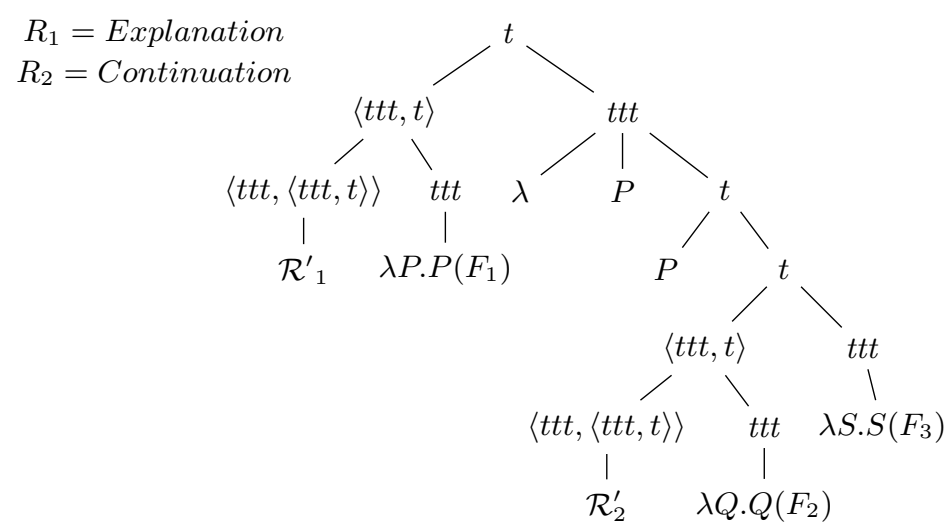

Fig. 8. Semantic derived tree for (1a) with interpretation $R_{1}\left(F_{1}, R_{2}\left(F_{2}, F_{3}\right)\right)$

We go on with (1b) with $\beta_{2}=\epsilon \div$ Explanation, for which the discourse structure is Explanation $\left(F_{1}, F_{2}\right) \wedge \operatorname{Explanation}\left(F_{2}, F_{3}\right)$ with a conjunction of formulae. This is obtained by adjoining $\beta_{2}$ at link (2) of $\beta_{1}$. The node with link (2) in the semantic tree of $\beta_{1}$ is of type $t t t$. Therefore, one must use tree (B) anchored by $\mathcal{R}_{2}^{\prime \prime}$, whose foot node is of type $t t t$. The semantic derived tree for (1b) is given in Fig. 9-a. The sub-tree rooted at Gorn address 2 results in $\lambda P .\left(\right.$ Explanation $\left.\left(F_{2}, F_{3}\right) \wedge P\left(F_{2}\right)\right)$ with $P:\langle t, t\rangle$. As only $F_{2}$ is under $P$, it is the second argument of $R_{1}=$ Explanation, whose first argument is $F_{1}$, hence the formula Explanation $\left(F_{1}, F_{2}\right) \wedge \operatorname{Explanation}\left(F_{2}, F_{3}\right)$. 
For (1c) with $\beta_{2}=$ then $\div$ Narration, the structure is Explanation $\left(F_{1}, F_{2}\right) \wedge$ $\operatorname{Narration}\left(F_{1}, F_{3}\right)$ with also a conjunction of formulae. This is obtained by adjoining $\beta_{2}$ at link (3) of $\beta_{1}$. This case is similar to the previous one, so we simply give the semantic derived tree in Fig. 9-b.

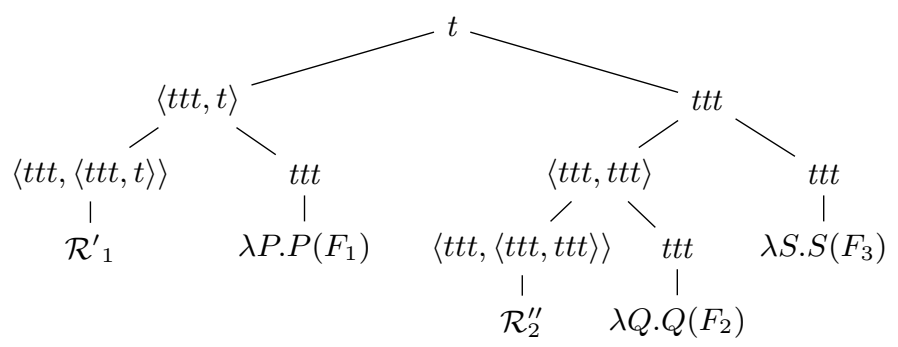

(a) (1b) with $R_{1}=R_{2}=$ Explanation

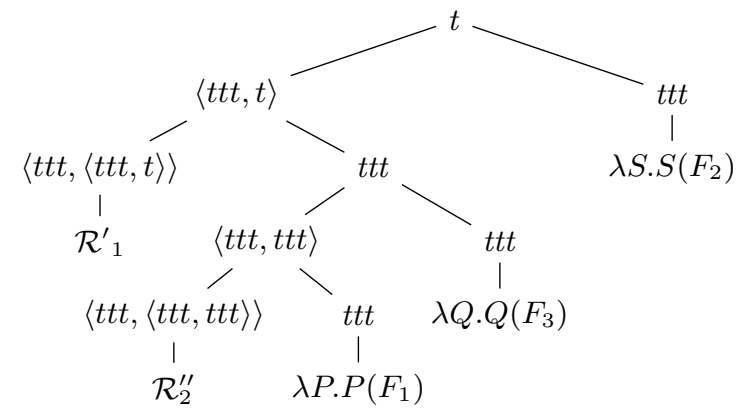

(b) (1c) with $R_{1}=$ Explanation and $R_{2}=$ Narration

Fig. 9. Semantic derived trees for (1b) and (1c) with interpretations $R_{1}\left(F_{1}, F_{2}\right) \wedge$ $R_{2}\left(F_{2}, F_{3}\right)$ and $R_{1}\left(F_{1}, F_{2}\right) \wedge R_{2}\left(F_{1}, F_{3}\right)$

Let us finish with (1d) with $\beta_{2}=\epsilon \div$ Commentary, for which the structure is Commentary $\left(\operatorname{Explanation}\left(F_{1}, F_{2}\right), F_{3}\right)$. This is obtained by adjoining $\beta_{2}$ at link (4) of $\beta_{1}$. The node with link (4) in the semantic tree of $\beta_{1}$ is of type $t$. Therefore, one must use tree $(\mathrm{A})$ anchored by $\mathcal{R}_{b}^{\prime}$. The semantic derived tree for (1d) is given in Fig. 10. The sub-tree rooted at Gorn address 1.2 results in $\lambda P . P\left(\operatorname{Explanation}\left(F_{1}, F_{2}\right)\right)$ with $P:\langle t, t\rangle$.

In conclusion, the four types of interpretation of DNFs of the form $C_{1} C_{o n j} C_{2}$. $A d v_{2} C_{3}$ are computed thanks to the four adjunction sites on the right frontier of the syntactic tree for $C_{1} C_{o n j} C_{2}$ and to semantic trees (A) and (B) whose foot nodes are respectively of type $t$ et $t t t$ and which are anchored by $\mathcal{R}_{i}^{\prime}$ and $\mathcal{R}_{i}^{\prime \prime}$.

For DNFs with three clauses of the form $C_{1} C_{o n n} C_{2} C_{o n n} C_{3}$, we have just examined the case $C_{1} C_{o n j_{1}} C_{2} . A d v_{2} C_{3}$ where $C o n n_{1}$ is a postposed conjunction and $C o n n_{2}$ an adverbial. Three cases are left: $C o n n_{1}$ is a postposed 


$$
\begin{gathered}
R_{1}=\text { Explanation } \\
R_{2}=\text { Commentary }
\end{gathered}
$$

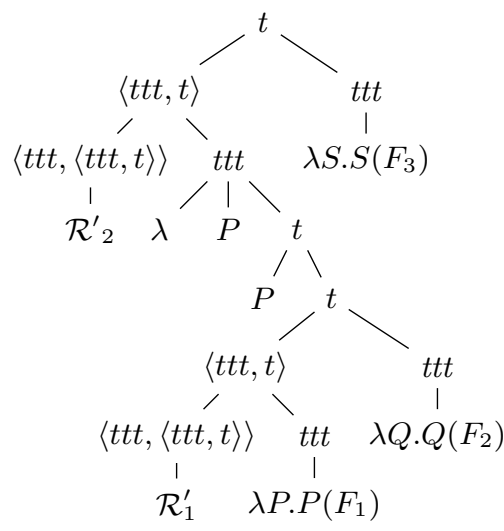

Fig. 10. Semantic derived trees for (1d) with the interpretation $R_{2}\left(R_{1}\left(F_{1}, F_{2}\right), F_{3}\right)$

conjunction and Conn 2 also, Conn 1 is an adverbial and Conn 2 also, Conn 1 is an adverbial and $\mathrm{Conn}_{2}$ a postposed conjunction. The first two cases raise no new issues. The third case, which concerns DNFs of the form $C_{1}$. Adv $C_{2} C_{2} \operatorname{conj}_{2} C_{3}$, raises the issue of fully implementing Constraint 3 from Sect. 2, which states that the mate segment of a postposed conjunction cannot cross a sentence boundary. This constraint is implemented thanks to features $[c o n j-p o s t= \pm]$ which decorate some nodes in the syntactic trees anchored by an adverbial or a postposed conjunction, which are given in Fig. 5. More precisely:

- the foot node of a tree anchored by a postposed conjunction is decorated with the top feature conj - post $=+$,

- the nodes with links (3) and (4) in a tree anchored by an adverbial are decorated with the bottom feature conj - post $=-$.

These features block the adjunction of $C o n j_{2} \div R_{2}$ at links (3) and (4) of $A d v_{1} \div R_{1}$ thanks to the unification failure $($ conj - post $=+) \cup($ conj - post $=-)$. Therefore, Conj $_{2} \div R_{2}$ can only adjoin at link (2) of $A d v_{1} \div R_{1}$ and at link (1) of $\tau_{2}^{\prime}$, which results respectively in the interpretations $R_{1}\left(F_{1}, F_{2}\right) \wedge R_{2}\left(F_{2}, F_{3}\right)$ and $R_{1}\left(F_{1}, R_{2}\left(F_{2}, F_{3}\right)\right)$. These interpretations conform to Constraint 3: the mate argument of $R_{2}$ is $F_{2}$, so the mate segment of $\mathrm{Conj}_{2}$ is $C_{2}$, which doesn't cross a sentence boundary.

Analysis of DNFs with $n$ clauses $(n>3)$ of the form $C_{1}$ Conn $_{1} C_{2} \ldots C_{n}$. For DNFs with $n$ clauses, no new mechanism is involved. Attaching the new segment Conn $_{n} C_{n+1}$ consists in adjoining Conn $_{n} \div R_{n}$ - in which $\tau_{n+1}^{\prime}$ is substituted - at link (1) of $\tau_{n}^{\prime}$ or at link (i) with $i \in\{2,3,4\}$ of a node $\beta_{k}=C o n n_{k} \div R_{k}$ in the derivation tree representing $C_{1} \ldots C_{n}$, the node at link (i) coming from the syntactic tree of $\beta_{k}$ being on the right frontier of the syntactic tree for $C_{1} \ldots C_{n}$ (to keep to the linear order of the DNF). As it is long and tedious to determine (the right frontier of) the derived syntactic tree, it is convenient to define a notion 
of right frontier on the derivation tree. Since derivation trees are intrinsically not ordered, a graphical convention which represents derivation trees as ordered must be called upon. The following convention is satisfactory: the nodes labeled $\tau_{k}^{\prime}$ projected onto a line are ordered by following the linear order of the DNF. With this order relation noted $\prec$, the nodes $\beta_{k}$ in the derivation tree which are possible sites of adjunction for a new segment are those on the right frontier of the derivation tree, while observing Constraint 5 which governs two adjunctions at links $\left(n\right.$ and $\left(m\right.$ of the same node: ${ }^{4}$

Constraint 5 If $\beta_{j}$ - in which $\tau_{j+1}^{\prime}$ is substituted - adjoins at link (n) of a node $\beta_{i}$, then $\beta_{k}$ - in which $\tau_{k+1}^{\prime}$ is substituted - can adjoin at link $m$ of the same node $\beta_{i}$ only if the following rule is observed: $\tau_{j+1}^{\prime} \prec \tau_{k+1}^{\prime} \Rightarrow n<m$ (with $n$ and $m$ belonging to $\{2,3,4\}$ ).

This constraint generalizes the one we formulated before, namely, if an adjunction is performed at node $D U(3)$ of a syntactic tree anchored by $C o n n_{i}$, then a new adjunction in this tree can be performed at $D U(4)$ but not at $D U(2$.

Implementation of RFC. The Right Frontier Constraint (RFC) postulated in SDRT relies on a distinction between two types of discourse relations: coordinating (Narration, Continuation) versus subordinating (Explanation, Commentary) ones. RFC says that it is forbidden to attach new information to the first argument of a coordinating relation [2]. As a consequence, for example, the interpretation $R_{1}\left(F_{1}, F_{2}\right) \wedge R_{2}\left(F_{1}, F_{3}\right)$ is excluded when $R_{1}$ is coordinating.

Implementing RFC in D-STAG requires first to distinguish the semantic trees anchored by a coordinating versus subordinating relation. This is achieved by creating two copies of semantic trees (A) and (B), which differ in a top feature $[$ coord $= \pm$ ] decorating their foot node. Then RFC is implemented by forbidding any adjunction at link (3) of the copies of (A) and (B) whose foot node is decorated with the feature $[$ coord $=+]$ (then, for example, the interpretation $R_{1}\left(F_{1}, F_{2}\right) \wedge R_{2}\left(F_{1}, F_{3}\right)$ is excluded when $R_{1}$ is coordinating).

SDRT postulates other semantic constraints based on the distinction between coordinating versus subordinating relations, for example the "Continuous Discourse Pattern" [18]. There is no room to describe them, however we can say that they are easily implemented in D-STAG thanks to the addition of a set of features in the semantic trees.

5.1.2 Preposed Conjunctions The syntactic tree anchored by a preposed conjunction is given in Fig. 11. It is designed so as to respect Constraints 1 and 4 given in Sect. 2. It differs from the syntactic trees anchored by an adverbial or a postposed conjunction, among other things, by the fact that the foot node $D U *$ is dominated by a node $D U$ with link (5). To take into account this new link, a node $t(5)$ dominating the foot node $t *$ is added into the two copies of semantic tree (A).

\footnotetext{
${ }^{4}$ This constraint is valid because we assigned links (2), (3) and (4) in a well-thought-out manner.
} 

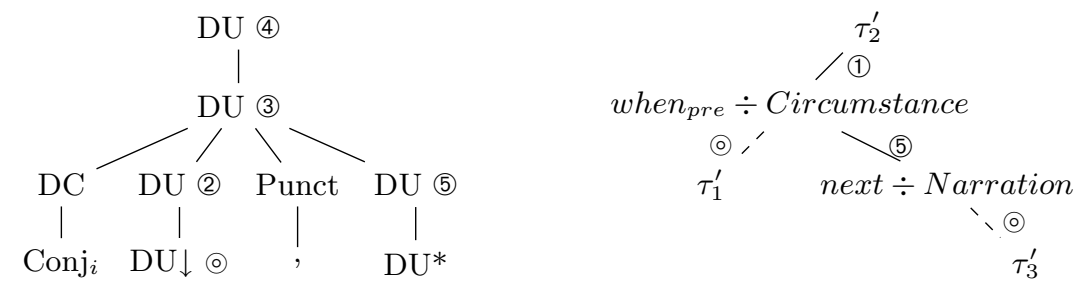

Fig. 11. Tree anchored by a preposed conjunction and derivation tree for (3)

An adjunction to link (5) is used when the preposed conjunction plays the role of a "framing adverbial" [19] as illustrated in (3) of the form When $C_{1}, C_{2}$. Next $C_{3}$. In this discourse, the preposed conjunction when - the framing adverbial has its mate segment which crosses a sentence boundary. Its interpretation is Circumstance (Narration $\left(F_{2}, F_{3}\right), F_{1}$ ), assuming that when conveys Circumstance and next conveys Narration.

(3) When he was in Paris, Fred went to the Eiffel Tower. Next, he visited The Louvre.

The derivation tree for (3) is given in Fig. 11. The semantic trees anchored by Circumstance and Narration are both (A). The arguments of $\mathcal{R}_{1}^{\prime}$ with $R_{1}=$ Circumstance are $\lambda P . P\left(\operatorname{Narration}\left(F_{2}, F_{3}\right)\right)$ and $\lambda Q . Q\left(F_{1}\right)$. Therefore, $\operatorname{Narration}\left(F_{2}, F_{3}\right)$ is the first argument of Circumstance, $F_{1}$ the second one. ${ }^{5}$

\subsection{STAG Grammar for Modifiers of Discourse Connectives/Relations}

As far as we are aware, modification of discourse connectives/relations is a phenomenon which has been neglected, even in SDRT. However, it is a common phenomenon as illustrated in (4).

(4)a. Fred is in a bad mood only/even/except when it is sunny.

b. You shouldn't trust John because, for example, he never returns what he borrows. [21]

c. John just broke his arm. So, for example, he can't cycle to work.[21].

In [21], for example is not considered as a connective modifier but as a connective whose interpretation is "parasitic" on the relation conveyed by the connective on its left. This position, which is not linguistically justified, leads to

\footnotetext{
${ }^{5}$ Examples such as (3) make it that, despite appearances, the syntactic discursive grammar of D-STAG is not a TIG (Tree Insertion Grammar) [20], since the right tree anchored by next is adjoined on the spine of the left tree anchored by the preposed conjunction when.
} 
laborious computations in D-LTAG [8, pp 31-35] to obtain the interpretation of (4b). On the contrary, in D-STAG, we propose that for example in (4b) or (4c) is a modifier of the connective on its left in the same way that only, even and except in (4a) are modifiers of the connective on their right. This position sounds more justified on linguistic grounds and it allows us to obtain the interpretation of a discourse such as (4b) in a very simple way, as we are going to show.

In D-STAG, connective modifiers anchor (syntactic) auxiliary trees whose foot node is $D C$ (left tree for only, even and except, right tree for for example). To adjoin these trees, we mark the node labelled $D C$ with link (6) in the syntactic trees for connectives (Fig. 5 and 11). At the semantic level, we assume that the contribution of a discourse relation modifier consists in transforming a functor $\mathcal{R}_{i}$ of type $\langle t,\langle t, t\rangle\rangle$ into another functor of the same type. Therefore, the nodes dominating $\mathcal{R}_{i}$ are marked with link (6) in the two copies of (A) and (B). Let us illustrate adjunctions to link (6) with (4b) of the form $C_{1}$ because for example $C_{2}$. As explained in [21], the interpretation of (4b) is Exemplification $\left(F_{2}, \lambda r\right.$.Explanation $\left.\left(F_{1}, r\right)\right)$ with $r: t$. To get this interpretation, we define the functor $\mathcal{F}$ or-ex as below. The pair named $\beta$ for-ex is given in Fig. 12, which also shows the derivation tree for $(4 \mathrm{~b})$. The functor $\Phi^{\prime}\left(\mathcal{F}\right.$ or-ex $\left.\left(\mathcal{R}_{i}\right)\right)$ with $R_{i}=$ Explanation results in the right interpretation.

Definition $3 \mathcal{F}$ or-ex $=\lambda \mathcal{R}_{i} p q$.Exemplification $\left(q, \lambda r . \mathcal{R}_{i}(p, r)\right)$ with $\mathcal{R}_{i}:\langle t,\langle t, t\rangle\rangle$ and $p, q: t$.
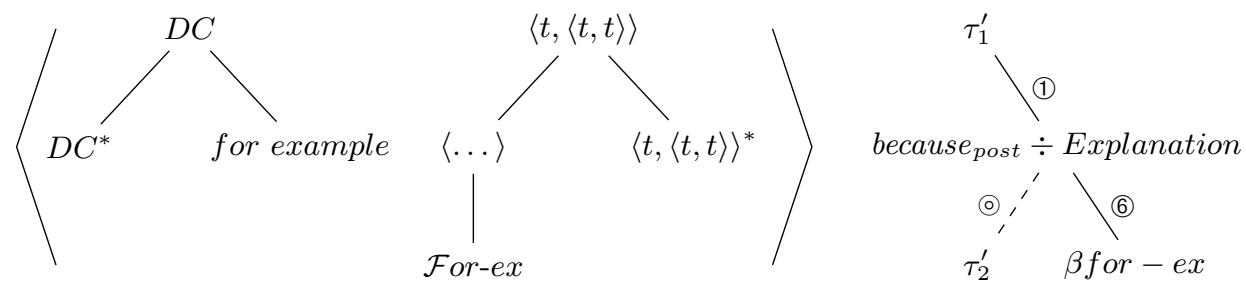

Fig. 12. Pair $\beta$ for-ex and derivation tree for (4b)

The correlative constructions with neither ... nor, either ... or, not only ... but also that are illustrated in (5) can easily be handled in D-STAG by considering e.g. neither and nor as (adverbial) modifiers of the subordinating conjunctions on their right. The fact that neither and nor cannot appear independently is taken into account by a set of features in the syntactic trees for connectives; however, there is no room to explain these features. For (5a) of the form $C_{1}$ neither when $C_{2}$ nor when $C_{3}$, the interpretation $\neg$ Condition $\left(F_{1}, F_{2}\right) \wedge$ $\neg$ Condition $\left(F_{1}, F_{3}\right)$ - assuming that when lexicalizes the relation Condition - is obtained (through an adjunction at link (3) by giving neither and nor the semantics of negation. For (5b) of the form $C_{1}$ either if $C_{2}$ or if $C_{3}$, the interpretation Condition $\left(F_{1}, F_{2}\right) \vee \operatorname{Condition}\left(F_{1}, F_{3}\right)=\neg\left(\neg \operatorname{Condition}\left(F_{1}, F_{2}\right) \wedge\right.$ 
$\neg$ Condition $\left.\left(F_{1}, F_{3}\right)\right)$ is obtained by a semantic tree associated to either with two parts, one for the local scope of negation, and the other one for the global scope of negation over the conjunction of formulae. ${ }^{6}$

(5)a. Fred is pleased neither when it is sunny nor when it is rainy.

b. Fred will come either if it is sunny or if it is rainy.

c. Fred is pleased not only when it is sunny but also when it is rainy.

\section{Comparison between D-STAG and D-LTAG}

D-STAG and D-LTAG [8] roughly share the same goal and the same architecture. The discrepancies between these two formalisms mainly lie in the discursive component. ${ }^{7}$ First, D-LTAG makes little use of discourse relations and ignores the distinction between coordinating versus subordinating relations. In short, it doesn't build on discourse theories. This is even a principle, as shown in the quotation [8, p 1] " D-LTAG presents a model of low-level discourse structure and interpretation that exploits the same mechanisms used at the sentence level and builds them directly on top of clause structure and interpretation". This prevents D-LTAG from taking advantage of the insights provided by discourse theories, which supply rhetorical knowledge, among other things.

Next, discourse connectives in D-STAG all anchor elementary trees with two arguments, whereas, in D-LTAG, most adverbial connectives (but not the empty one $\epsilon$ ) anchor trees with only one argument (this is, for example, the case for then whose host segment is "structurally" provided, the mate segment being "anaphorically" provided [21]). Moreover, subordinating conjunctions in D-LTAG anchor trees with two arguments, but these trees are initial trees whereas they are auxiliary in D-STAG (subordinating conjunctions are considered in D-LTAG as "structural" connectives, neglecting the fact that one of their segments can cross a sentence boundary). These discrepancies between the trees anchored by a connective lead to crucial differences in the discursive analyses, especially in the semantic ones. As an illustration, the syntactic tree and derivation tree produced by D-LTAG for (1c) are given in Fig. 13.

The syntactic tree includes three nodes labelled $D C$, while it includes only two nodes labelled $D C$ in D-STAG since the empty connective $\epsilon$ is introduced only in the absence of any other adverbial. The derivation tree results in the discourse structure Narration (Explanation $\left.\left(F_{1}, F_{2}\right), F_{3}\right)$, which is wrong: the explanation given for Fred's visit to the supermarket (his fridge was empty) shouldn't be under the scope of Narration, which is a relation linking together two events and not a causal relation and an event. As explained before, the discourse structure for (1c) is Explanation $\left(F_{1}, F_{2}\right) \wedge \operatorname{Narration}\left(F_{1}, F_{3}\right)$. This

\footnotetext{
${ }^{6}$ This is the only case which requires a multi-component semantic tree (for the discourses we have dealt with so far).

7 The sentential components of D-STAG and D-LTAG are also crucially different, since D-STAG relies on STAG, which is not the case for D-LTAG. However, the sentential level is out of the scope of this paper, see [6] for a discussion on the various approaches for a sentential syntax-semantics interface.
} 
structure, which corresponds to a non tree-shaped dependency graph, cannot be obtained in D-LTAG.
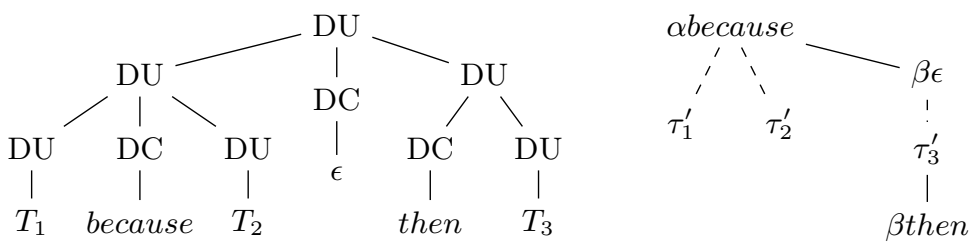

Fig. 13. D-LTAG syntactic tree and derivation tree for (1c)

"The distinction between structural and anaphoric connectives in D-LTAG is based on considerations of computational economy and behavioral evidence from cases of multiple connectives" [21, p 552]. In (6), whose DNF is of the form $C_{1}$ but $C_{2}$ because then $C_{3}$, two connectives share the same host clause $C_{3}$.

(6) John ordered three cases of Barolo. But he had to cancel the order because then he discovered he was broke. [21]

In D-STAG, we propose the following solution to handle multiple connectives. The DNF for (6) is automatically converted into $C_{1}$ but $C_{2}$ because $\overline{C_{3}}$ then $C_{3}$ which conforms to the regular pattern for DNFs (without any preposed conjunction). The tree pair $\overline{\tau_{3}^{\prime}}$ associated to $\overline{C_{3}}$ is given in Fig. 14-a: the syntactic leaf is the empty string $\epsilon$, the semantic leaf is $F_{3}$ (the semantic formula for $C_{3}$ ). So we can say that $\overline{C_{3}}$ is the (fake) host clause of because, which has only a semantic contribution. The interpretation of (6), i.e. Contrast $\left(F_{1}, F_{2}\right) \wedge \operatorname{Explanation}\left(F_{2}, F_{3}\right) \wedge \operatorname{After}\left(F_{1}, F_{3}\right)$ assuming that then conveys After, is obtained by the mechanisms described in Sect. 5.1.1 which build the derivation tree given in Fig. 14-b (with $\beta_{1}=$ but $\div$ Contrast, $\beta_{2}=$ because $\div$ Explanation and $\beta_{3}=$ then $\div$ After $)$.
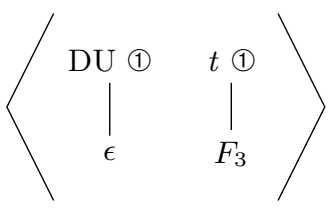

(a) $\overline{\tau_{3}^{\prime}}$

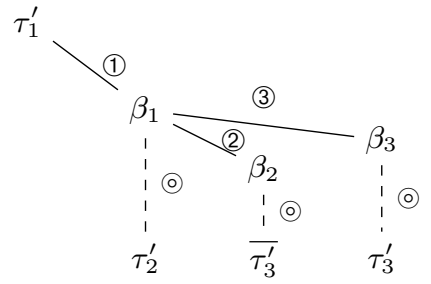

(b) Derivation tree for (6)

Fig. 14. Tree pair $\overline{\tau_{3}^{\prime}}$ and derivation tree for (6) 


\section{Conclusion}

We have presented D-STAG, a formalism which extends a sentential syntaxsemantics interface to the discourse level. It allows us to compute discourse structures which are compatible with those produced in SDRT, an elaborated discourse theory. The STAG discursive grammar mainly consists of syntactic trees anchored by a connective which are paired with semantic trees anchored by the discourse relation (or one of the discourse relations) lexicalized by the connective. It is designed so that discourse structures correspond to dependency graphs which are not necessarily tree shaped. The STAG discursive grammar also includes trees anchored by modifiers of discourse connectives/relations.

The discursive component takes as input a DNF that is computed by a sentencediscourse interface. This interface is neccesary if one doesn't want to make any change to the sentential analyzer. A DNF is a sequence of discourse words which follows a regular grammar. The regular grammar we have presented in this paper is not yet completed. We plan to extend it, especially to take into account those sub-clauses which play a role at the discursive level. This extended regular grammar will lead to an extended STAG discursive grammar. We don't foresee any crucial difficulty for this grammar, thanks to STAG's richness of expressivity.

The implementation of D-STAG in a French discourse analyzer is work in progress [22]. The analyzer will produce a forest of dependency trees which represents the set of possible analyses. The extraction of the best analysis (or the $n$ best analyses) will require to build probabilistic disambiguation models based on the French annotated corpus Annodis [23]. ${ }^{8}$

\section{References}

1. Asher, N.: Reference to Abstract Objects in Discourse. Kluwer, Dordrecht (1993)

2. Asher, N., Lascarides, A.: Logics of Conversation. Cambridge University Press, Cambridge (2003)

3. Joshi, A.: Tree-adjoining grammars. In Dowty, D., Karttunen, L., Zwicky, A., eds.: Natural language parsing. Cambridge University Press (1985) 206-250

4. Shieber, S.: Restricting the weak-generative capacity of synchronous tree-adjoining grammars. Computational Intelligence 10(4) (1994) 371-385

5. Shieber, S., Schabes, Y.: Synchronous tree-adjoining grammars. In: Proceedings of the 13th International Conference on Computational Linguistics. Volume 3., Helsinki (1990) 253-258

6. Nesson, R., Shieber, S.: Simpler TAG semantics through synchronization. In: Formal Grammars, Malaga (2006)

7. Danlos, L.: G-TAG: A lexicalized formalism for text generation inspired from TAG. In Abeill, A., Rambow, O., eds.: TAG Grammar. CSLI (2001)

8. Forbes-Riley, K., Webber, B., Joshi, A.: Computing discourse semantics: The predicate-argument semantics of discourse connectives in D-LTAG. Journal of Semantics 23(1) (2006)

\footnotetext{
${ }^{8}$ http://w3.erss.univ-tlse2.fr:8080/index.jsp?perso=annodis\&subURL= ANNODISfr.html
} 
9. Harris, Z.: Mathematical Structures of Language. Krieger Pub co, New York (1986)

10. Mann, W.C., Thompson, S.A.: Rhetorical structure theory : Toward a functional theory of text organization. Text 8(3) (1988) 243-281

11. Marcu, D.: The rhetorical parsing of unrestricted texts: A surface-based approach. Computational Linguistics 26(3) (2000) 395-448

12. Wolf, F., Gibson, E.: Coherence in Natural Language: Data Structures and Applications. The MIT Press, London (2006)

13. Danlos, L.: Discourse dependency structures as constrained DAGs. In: Proceedings of SIGDIAL'04, Boston (2004) 127-135

14. Danlos, L.: Strong generative capacity of RST, SDRT and discourse dependency DAGs. In Benz, A., Kühnlein, P., eds.: Constraints in Discourse. Benjamins (2007) 69-95

15. Lee, A., Prasad, R., Joshi, A., Webber, B.: Departures from tree structures in discourse: Shared arguments in the Penn Discourse Tree Bank. In: Proceedings of the Constraints in Discourse Workshop (CID'08), Postdam, Germany (2008)

16. PDTB Group: The Penn Discourse Treebank 2.0 annotation manual. Technical report, Institute for Research in Cognitive Science, University of Philadelphia (2008)

17. Marcu, D.: The Theory and Practice of Discourse Parsing and Summarization. The MIT Press (2000)

18. Asher, N., Vieu, L.: Subordinating and coordinating discourse relations. Lingua 115(4) (2005) 591-610

19. Charolles, M.: Framing adverbials and their role in discourse cohesion. In: Proceedings of SEM-05, Biarritz (2005) 194-201

20. Schabes, Y., Waters, R.: Tree Insertion Grammar. Computational Intelligence 21 (1995) 479-514

21. Webber, B., Joshi, A., Stone, M., Knott, A.: Anaphora and discourse structure. Computational Linguistics 29(4) (2003) 545-587

22. Danlos, L.: D-STAG : un formalisme d'analyse automatique de discours basé sur les TAG synchrones. Revue TAL 50 (2009) 1-31

23. Péry-Woodley, M.P., Asher, N., Enjalbert, P.: ANNODIS: une approche outillée de l'annotation de structures discursives. In: Proceedings of TALN'09, Senlis, France (2009) 190-196 